\title{
Independent Coordinate Coupling Method for Free Vibration Analysis of a Plate With Holes
}

\author{
Moon Kyu Kwak and Seok Heo \\ Dongguk University \\ Republic of Korea
}

\section{Introduction}

A rectangular plate with a rectangular or a circular hole has been widely used as a substructure for ship, airplane, and plant. Uniform circular and annular plates have been also widely used as structural components for various industrial applications and their dynamic behaviors can be described by exact solutions. However, the vibration characteristics of a circular plate with an eccentric circular hole cannot be analyzed easily. The vibration characteristics of a rectangular plate with a hole can be solved by either the Rayleigh-Ritz method or the finite element method. The Rayleigh-Ritz method is an effective method when the rectangular plate has a rectangular hole. However, it cannot be easily applied to the case of a rectangular plate with a circular hole since the admissible functions for the rectangular hole domain do not permit closed-form integrals. The finite element method is a versatile tool for structural vibration analysis and therefore, can be applied to any of the cases mentioned above. But it does not permit qualitative analysis and requires enormous computational time.

Tremendous amount of research has been carried out on the free vibration of various problems involving various shape and method. Monahan et al.(1970) applied the finite element method to a clamped rectangular plate with a rectangular hole and verified the numerical results by experiments. Paramasivam(1973) used the finite difference method for a simply-supported and clamped rectangular plate with a rectangular hole. There are many research works concerning plate with a single hole but a few work on plate with multiple holes. Aksu and Ali(1976) also used the finite difference method to analyze a rectangular plate with more than two holes. Rajamani and Prabhakaran(1977) assumed that the effect of a hole is equivalent to an externally applied loading and carried out a numerical analysis based on this assumption for a composite plate. Rajamani and Prabhakaran(1977) investigated the effect of a hole on the natural vibration characteristics of isotropic and orthotropic plates with simply-supported and clamped boundary conditions. Ali and Atwal(1980) applied the Rayleigh-Ritz method to a simply-supported rectangular plate with a rectangular hole, using the static deflection curves for a uniform loading as admissible functions. Lam et al.(1989) divided the rectangular plate with a hole into several sub areas and applied the modified Rayleigh-Ritz method. Lam and Hung(1990) applied the same method to a stiffened plate. The admissible functions used in (Lam et al. 1989, Lam and Hung 1990) are the orthogonal polynomial functions proposed by Bhat $(1985,1990)$. Laura et al.(1997) calculated the natural vibration characteristics of a simply-supported rectangular 
plate with a rectangular hole by the classical Rayleigh-Ritz method. Sakiyama et al.(2003) analyzed the natural vibration characteristics of an orthotropic plate with a square hole by means of the Green function assuming the hole as an extremely thin plate.

The vibration analysis of a rectangular plate with a circular hole does not lend an easy approach since the geometry of the hole is not the same as the geometry of the rectangular plate. Takahashi(1958) used the classical Rayleigh-Ritz method after deriving the total energy by subtracting the energy of the hole from the energy of the whole plate. He employed the eigenfunctions of a uniform beam as admissible functions. Joga-Rao and Pickett(1961) proposed the use of algebraic polynomial functions and biharmonic singular functions. Kumai(1952), Hegarty(1975), Eastep and Hemmig(1978), and Nagaya(1951) used the point-matching method for the analysis of a rectangular plate with a circular hole. The point-matching method employed the polar coordinate system based on the circular hole and the boundary conditions were satisfied along the points located on the sides of the rectangular plate. Lee and Kim(1984) carried out vibration experiments on the rectangular plates with a hole in air and water. Kim et al.(1987) performed the theoretical analysis on a stiffened rectangular plate with a hole. Avalos and Laura(2003) calculated the natural frequency of a simply-supported rectangular plate with two rectangular holes using the classical Rayleigh-Ritz method. Lee et al.(1994) analyzed a square plate with two collinear circular holes using the classical Rayleigh-Ritz method.

A circular plate with en eccentric circular hole has been treated by various methods. Nagaya(1980) developed an analytical method which utilizes a coordinate system whose origin is at the center of the eccentric hole and an infinite series to represent the outer boundary curve. Khurasia and Rawtani(1978) studied the effect of the eccentricity of the hole on the vibration characteristics of the circular plate by using the triangular finite element method. Lin(1982) used an analytical method based on the transformation of Bessel functions to calculate the free transverse vibrations of uniform circular plates and membranes with eccentric holes. Laura et al.(2006) applied the Rayleigh-Ritz method to circular plates restrained against rotation with an eccentric circular perforation with a free edge. Cheng et al.(2003) used the finite element analysis code, Nastran, to analyze the effects of the hole eccentricity, hole size and boundary condition on the vibration modes of annular-like plates. Lee et al.(2007) used an indirect formulation in conjunction with degenerate kernels and Fourier series to solve for the natural frequencies and modes of circular plates with multiple circular holes and verified the finite element solution by using ABAQUS. Zhong and $\mathrm{Yu}(2007)$ formulated a weak-form quadrature element method to study the flexural vibrations of an eccentric annular Mindlin plate.

Recently, Kwak et al.(2005, 2006, 2007), and Heo and Kwak(2008) presented a new method called the Independent Coordinate Coupling Method(ICCM) for the free vibration analysis of a rectangular plate with a rectangular or a circular hole. This method utilizes independent coordinates for the global and local domains and the transformation matrix between the local and global coordinates which is obtained by imposing a kinematical relation on the displacement matching condition inside the hole domain. In the Rayleigh-Ritz method, the effect of the hole can be considered by the subtraction of the energy for the hole domain in deriving the total energy. In doing so, the previous researches considered only the global coordinate system for the integration. The ICCM is advantageous because it does not need to use a complex integration process to determine the total energy of the plate with a hole. The ICCM can be also applied to a circular plate with an eccentric hole. The numerical results obtained by the ICCM were compared to the numerical results of the classical 
approach, the finite element method, and the experimental results. The numerical results show the efficacy of the proposed method.

\section{Rayleigh-Ritz method for free vibration analysis of rectangular plate}

Let us consider a rectangular plate with side lengths $a$ in the $X$ direction and $b$ in the $Y$ direction. The kinetic and potential energies of the rectangular plate can be expressed as

$$
\begin{gathered}
T_{R}=\frac{1}{2} \rho h \int_{0}^{a} \int_{0}^{b} \dot{w}_{r}^{2} d x d y \\
V_{R}=\frac{1}{2} D \int_{0}^{a} \int_{0}^{b}\left[\left(\frac{\partial^{2} w_{r}}{\partial x^{2}}\right)^{2}+\left(\frac{\partial^{2} w_{r}}{\partial y^{2}}\right)^{2}+2 v\left(\frac{\partial^{2} w_{r}}{\partial x^{2}} \frac{\partial^{2} w_{r}}{\partial y^{2}}\right)+2(1-v)\left(\frac{\partial^{2} w_{r}}{\partial x \partial y}\right)^{2}\right] d x d y
\end{gathered}
$$

where $w_{r}=w_{r}(x, y, t)$ represents the deflection of the plate, $\rho$ the mass density, $h$ the thickness, $D=E h^{3} / 12\left(1-v^{2}\right), E$ the Young's modulus, and $v$ the Poisson's ratio.

By using the non-dimensional variables, $\xi=x / a, \eta=y / b$ and the assumed mode method, the deflection of the plate can be expressed as

$$
w_{r}(\xi, \eta, t)=\Phi_{r}(\xi, \eta) q_{r}(t)
$$

where $\Phi_{r}(\xi, \eta)=\left[\Phi_{r 1} \Phi_{r 2} \ldots \Phi_{r m}\right]$ is a $1 \times m$ matrix consisting of the admissible functions and $q_{r}(t)=\left[q_{r 1} q_{r 2} \ldots q_{r m}\right]^{T}$ is a $m \times 1$ vector consisting of generalized coordinates, in which $m$ is the number of admissible functions used for the approximation of the deflection. Inserting Eq. (3) into Eqs. (1) and (2) results in Eq. (4).

$$
T_{R}=\frac{1}{2} \dot{q}_{r}^{T} M_{r} \dot{q}_{r}, \quad V_{R}=\frac{1}{2} q_{r}^{T} K_{r} q_{r}
$$

where

In which

$$
M_{r}=\rho h a b \bar{M}_{r}, \quad K_{r}=\frac{D b}{a^{3}} \bar{K}_{r}
$$

$$
\begin{gathered}
\bar{M}_{r}=\int_{0}^{1} \int_{0}^{1} \Phi_{r}^{T} \Phi_{r} d \xi d \eta \\
\bar{K}_{r}=\int_{0}^{1} \int_{0}^{1}\left[\frac{\partial^{2} \Phi_{r}^{T}}{\partial \xi^{2}} \frac{\partial^{2} \Phi_{r}}{\partial \xi^{2}}+\alpha^{4} \frac{\partial^{2} \Phi_{r}^{T}}{\partial \eta^{2}} \frac{\partial^{2} \Phi_{r}}{\partial \eta^{2}}+v \alpha^{2}\left(\frac{\partial^{2} \Phi_{r}^{T}}{\partial \xi^{2}} \frac{\partial^{2} \Phi_{r}}{\partial \eta^{2}}+\frac{\partial^{2} \Phi_{r}^{T}}{\partial \eta^{2}} \frac{\partial^{2} \Phi_{r}}{\partial \xi^{2}}\right)\right. \\
\left.+2(1-v) \alpha^{2} \frac{\partial^{2} \Phi_{r}^{T}}{\partial \xi \partial \eta} \frac{\partial^{2} \Phi_{r}}{\partial \xi \partial \eta}\right] d \xi d \eta
\end{gathered}
$$

$\bar{M}_{r}, \bar{K}_{r}$ represent the non-dimensionalized mass and stiffness matrices, respectively, and $\alpha=a / b$ represents the aspect ratio of the plate. The equation of motion can be derived by inserting Eq. (4) into the Lagrange's equation and the eigenvalue problem can be expressed as 


$$
\left[K_{r}-\omega^{2} M_{r}\right] A=0
$$

If we use the non-dimensionalized mass and stiffness matrices introduced in Eq. (5), the eigenvalue problem given by Eq. (7) can be also non-dimensionalized.

$$
\left[\bar{K}_{r}-\bar{\omega}^{2} \bar{M}_{r}\right] A=0
$$

where $\bar{\omega}$ is the non-dimensionalized natural frequency, which has the relationship with the natural frequency as follows:

$$
\bar{\omega}=\omega \sqrt{\frac{\rho h a^{4}}{D}}
$$

To calculate the mass and stiffness matrices given by Eq. (6) easily, the admissible function matrix given by Eq. (3) needs to be expressed in terms of admissible function matrices in each direction.

$$
\Phi_{r i}(\xi, \eta)=\phi_{i}(\xi) \psi_{i}(\eta), i=1,2, \ldots, m
$$

Then, the non-dimensionalized mass and stiffness matrices given by Eq. (6) can be expressed as [Kwak and $\operatorname{Han}(2007)]$

$$
\begin{gathered}
\left(\bar{M}_{r}\right)_{i j}=X_{i j} Y_{i j} \\
\left(\bar{K}_{r}\right)_{i j}=\hat{X}_{i j} Y_{i j}+\alpha^{4} X_{i j} \hat{Y}_{i j}+\alpha^{2} v\left(\tilde{X}_{j i} \tilde{Y}_{i j}+\tilde{X}_{i j} \tilde{Y}_{j i}\right)+\alpha^{2}(1-v) \bar{X}_{i j} \bar{Y}_{i j}, \quad i, j=1,2, \ldots, m
\end{gathered}
$$

where

$$
\begin{gathered}
X_{i j}=\int_{0}^{1} \phi_{i} \phi_{j} d \xi, \bar{X}_{i j}=\int_{0}^{1} \phi_{i}^{\prime} \phi_{j}^{\prime} d \xi, \hat{X}_{i j}=\int_{0}^{1} \phi_{i}^{\prime \prime} \phi_{j}^{\prime \prime} d \xi, \tilde{X}_{i j}=\int_{0}^{1} \phi_{i} \phi_{j}^{\prime \prime} d \xi \\
Y_{i j}=\int_{0}^{1} \psi_{i} \psi_{j} d \eta, \bar{Y}_{i j}=\int_{0}^{1} \psi_{i}^{\prime} \psi_{j}^{\prime} d \eta, \hat{Y}_{i j}=\int_{0}^{1} \psi_{i}^{\prime \prime} \psi_{j}^{\prime \prime} d \eta, \tilde{Y}_{i j}=\int_{0}^{1} \psi_{i} \psi_{j}^{\prime \prime} d \eta, \quad i, j=1,2, \ldots, m
\end{gathered}
$$

If $n$ admissible functions are used in the $X$ and $Y$ directions and the combination of admissible functions are used, a total of $n^{2}$ admissible functions can be obtained, which yields $m=n^{2}$. If each type of admissible functions are considered as $\chi_{i}(i=1,2, \ldots, n)$ and $\gamma_{i}(i=1,2, \ldots, n)$, then the relationship of between the sequence of the admissible function introduced in Eq. (10) and those of separated admissible functions can be expressed as

$$
\phi_{k}=\left\{\begin{array}{ll}
\chi_{1} & 1 \leq k \leq n \\
\chi_{2} & n+1 \leq k \leq 2 n \\
\chi_{3} & 2 n+1 \leq k \leq 3 n \\
& \vdots \\
\chi_{n} & (n-1) n+1 \leq k \leq n^{2}
\end{array} \quad, \psi_{k}=\left\{\begin{array}{cc}
\gamma_{k} & 1 \leq k \leq n \\
\gamma_{k-n} & n+1 \leq k \leq 2 n \\
\gamma_{k-2 n} & 2 n+1 \leq k \leq 3 n \\
\vdots & \\
\gamma_{k-(n-1) n} & (n-1) n+1 \leq k \leq n^{2}
\end{array}\right.\right.
$$


Therefore, instead of integrating $m^{2}=n^{4}$ elements in Eq. (12), $n^{2}$ integrations and matrix rearrangement will suffice. First, let us calculate the following.

$$
\begin{gathered}
\Sigma_{i j}=\int_{0}^{1} \chi_{i} \chi_{j} d \xi, \bar{\Sigma}_{i j}=\int_{0}^{1} \chi_{i}^{\prime} \chi_{j}^{\prime} d \xi, \hat{\Sigma}_{i j}=\int_{0}^{1} \chi_{i}^{\prime \prime} \chi_{j}^{\prime \prime} d \xi, \tilde{\Sigma}_{i j}=\int_{0}^{1} \chi_{i} \chi_{j}^{\prime \prime} d \xi \\
\Gamma_{i j}=\int_{0}^{1} \gamma_{i} \gamma_{j} d \eta, \bar{\Gamma}_{i j}=\int_{0}^{1} \gamma_{i}^{\prime} \gamma_{j}^{\prime} d \eta \quad \hat{\Gamma}_{i j}=\int_{0}^{1} \gamma_{i}^{\prime \prime} \gamma_{j}^{\prime \prime} d \eta, \tilde{\Gamma}_{i j}=\int_{0}^{1} \gamma_{i} \gamma_{j}^{\prime \prime} d \eta, i, j=1,2, \ldots, n
\end{gathered}
$$

And then the matrices given by Eq. (12) can be derived as follows:

$$
\begin{gathered}
X=\left[\begin{array}{cccc}
\Sigma_{11} \bar{I} & \Sigma_{12} \bar{I} & \cdots & \Sigma_{1 n} \bar{I} \\
\Sigma_{21} \bar{I} & \Sigma_{22} \bar{I} & \cdots & \Sigma_{2 n} \bar{I} \\
\vdots & \vdots & \ddots & \vdots \\
\Sigma_{n 1} \bar{I} & \Sigma_{n 2} \bar{I} & \cdots & \Sigma_{n n} \bar{I}
\end{array}\right], \bar{X}=\left[\begin{array}{cccc}
\bar{\Sigma}_{11} \bar{I} & \bar{\Sigma}_{12} \bar{I} & \ldots & \bar{\Sigma}_{1 n} \bar{I} \\
\bar{\Sigma}_{21} \bar{I} & \bar{\Sigma}_{22} \bar{I} & \ldots & \bar{\Sigma}_{2 n} \bar{I} \\
\vdots & \vdots & \ddots & \vdots \\
\bar{\Sigma}_{n 1} \bar{I} & \bar{\Sigma}_{n 2} \bar{I} & \ldots & \bar{\Sigma}_{n n} \bar{I}
\end{array}\right] \\
\hat{X}=\left[\begin{array}{cccc}
\hat{\Sigma}_{11} \bar{I} & \hat{\Sigma}_{12} \bar{I} & \cdots & \hat{\Sigma}_{1 n} \bar{I} \\
\hat{\Sigma}_{21} \bar{I} & \hat{\Sigma}_{22} \bar{I} & \cdots & \hat{\Sigma}_{2 n} \bar{I} \\
\vdots & \vdots & \ddots & \vdots \\
\hat{\Sigma}_{n 1} \bar{I} & \hat{\Sigma}_{n 2} \bar{I} & \cdots & \hat{\Sigma}_{n n} \bar{I}
\end{array}\right], \quad \tilde{X}=\left[\begin{array}{cccc}
\tilde{\Sigma}_{11} \bar{I} & \tilde{\Sigma}_{12} \bar{I} & \cdots & \tilde{\Sigma}_{1 n} \bar{I} \\
\tilde{\Sigma}_{21} \bar{I} & \tilde{\Sigma}_{22} \bar{I} & \ldots & \tilde{\Sigma}_{2 n} \bar{I} \\
\vdots & \vdots & \ddots & \vdots \\
\tilde{\Sigma}_{n 1} \bar{I} & \tilde{\Sigma}_{n 2} \bar{I} & \cdots & \tilde{\Sigma}_{n n} \bar{I}
\end{array}\right] \\
Y=\left[\begin{array}{cccc}
\Gamma & \Gamma & \cdots & \Gamma \\
\Gamma & \Gamma & \cdots & \Gamma \\
\vdots & \vdots & \ddots & \vdots \\
\Gamma & \Gamma & \cdots & \Gamma
\end{array}\right], \quad \bar{Y}=\left[\begin{array}{cccc}
\bar{\Gamma} & \bar{\Gamma} & \cdots & \bar{\Gamma} \\
\bar{\Gamma} & \bar{\Gamma} & \cdots & \bar{\Gamma} \\
\vdots & \vdots & \ddots & \vdots \\
\bar{\Gamma} & \bar{\Gamma} & \cdots & \bar{\Gamma}
\end{array}\right], \\
\hat{Y}=\left[\begin{array}{cccc}
\hat{\Gamma} & \hat{\Gamma} & \cdots & \hat{\Gamma} \\
\hat{\Gamma} & \hat{\Gamma} & \cdots & \hat{\Gamma} \\
\vdots & \vdots & \ddots & \vdots \\
\hat{\Gamma} & \hat{\Gamma} & \cdots & \hat{\Gamma}
\end{array}\right], \hat{Y}=\left[\begin{array}{cccc}
\hat{\Gamma} & \hat{\Gamma} & \cdots & \hat{\Gamma} \\
\hat{\Gamma} & \hat{\Gamma} & \cdots & \hat{\Gamma} \\
\vdots & \vdots & \ddots & \vdots \\
\hat{\Gamma} & \hat{\Gamma} & \cdots & \hat{\Gamma}
\end{array}\right]
\end{gathered}
$$

where $\bar{I}$ is an $n \times n$ matrix full of ones.

Let us consider the simply-supported case in the $X$ direction. In this case, the eigenfunction of the uniform beam can be used as an admissible function.

$$
\chi_{i}=\sqrt{2} \sin i \pi \xi, \quad i=1,2, \ldots n
$$

In the case of the clamped condition in the $X$ direction, the eigenfunction of a clampedclamped uniform beam can be used.

$$
\chi_{i}=\cosh \lambda_{i} \xi-\cos \lambda_{i} \xi-\sigma_{i}\left(\sinh \lambda_{i} \xi-\sin \lambda_{i} \xi\right), \quad i=1,2, \ldots, n
$$

where $\lambda_{i}=4.730,7.853,10.996,14.137, \ldots$ and $\sigma_{i}=\left(\cosh \lambda_{i}-\cos \lambda_{i}\right) /\left(\sinh \lambda_{i}-\sin \lambda_{i}\right)$. In the case of a free-edge condition in the $X$ direction, we can use the eigenfunction of a free-free uniform beam. 


$$
\begin{gathered}
\chi_{1}=1, \chi_{2}=\sqrt{12}\left(\xi-\frac{1}{2}\right) \\
\chi_{i+2}=\cosh \lambda_{i} \xi+\cos \lambda_{i} \xi-\sigma_{i}\left(\sinh \lambda_{i} \xi+\sin \lambda_{i} \xi\right), \quad i=1,2, \ldots n-2
\end{gathered}
$$

where $\lambda_{i}$ and $\sigma_{i}$ are the same as the ones for the clamped-clamped beam, and the first and the second modes represent the rigid-body modes. $\Sigma_{i j}, \bar{\Sigma}_{i j}, \hat{\Sigma}_{i j}, \tilde{\Sigma}_{i j}$ for each case are given in the work of Kwak and Han(2007).

For the admissible functions in the $y$ direction, $\gamma_{i}$, the same method can be applied. The combination of different admissible functions can yield various boundary conditions.

\section{Rayleigh-Ritz method for free vibration analysis of circular plate}

Let us consider a uniform circular plate with radius, $R$, and thickness, $h$. The kinetic and potential energies can be expressed as follows:

$$
\begin{gathered}
T_{C}=\frac{1}{2} \rho h \int_{0}^{2 \pi} \int_{0}^{R} \dot{w}_{c}^{2} r d r d \theta \\
V_{C}=\frac{1}{2} D \int_{0}^{2 \pi} \int_{0}^{R}\left\{\left(\frac{\partial^{2} w_{c}}{\partial r^{2}}+\frac{1}{r} \frac{\partial w_{c}}{\partial r}+\frac{1}{r^{2}} \frac{\partial^{2} w_{c}}{\partial \theta^{2}}\right)^{2}-2(1-v)\left[\left(\frac{\partial^{2} w_{c}}{\partial r^{2}}\right)\left(\frac{1}{r} \frac{\partial w_{c}}{\partial r}+\frac{1}{r^{2}} \frac{\partial^{2} w_{c}}{\partial \theta^{2}}\right)\right.\right. \\
\left.\left.-\left(\frac{1}{r} \frac{\partial^{2} w_{c}}{\partial r \partial \theta}-\frac{1}{r^{2}} \frac{\partial^{2} w_{c}}{\partial \theta^{2}}\right)^{2}\right]\right\} r d r d \theta
\end{gathered}
$$

Unlike the uniform rectangular plate, simply-supported, clamped, and free-edge uniform circular plates have eigenfunctions. Hence, the deflection of the circular plate can be expressed as the combination of eigenfunctions and generalized coordinates.

$$
w_{c}(r, \theta, t)=\sum_{i=1}^{n_{c}} \Phi_{c i}(r, \theta) q_{c i}(t)=\Phi_{c}(r, \theta) q_{c}(t)
$$

where $\Phi_{c i}(r, \theta)$ represents the eigenfunction of the uniform circular plate and $q_{c i}(t)$ represents the generalized coordinate. Inserting Eq. (20) into Eq. (19) results in the following.

where

$$
T_{C}=\frac{1}{2} \dot{q}_{c}^{T} M_{c} \dot{q}_{c}, V_{C}=\frac{1}{2} q_{c}^{T} K_{c} q_{c}
$$

$$
M_{c}=\rho h \pi R^{2} I, \quad K_{c}=\frac{\pi D}{R^{2}} \Lambda_{c}
$$

in which $I$ is an $n_{c} \times n_{c}$ identity matrix, $\Lambda_{c}$ is an $n_{c} \times n_{c}$ diagonal matrix whose diagonals are $\lambda_{i}^{4}$. The eigenvalue has the expression, $\lambda^{4}=\omega^{2} \rho h R^{4} / D$.

Since our study is concerned with either a rectangular or a circular hole, we consider only a free-edge circular plate [Itao and Crandall(1979)]. If the eigenfunctions are rearranged in ascending order, we can have 


$$
\begin{gathered}
\Phi_{c 1}=1, \Phi_{c 2}=\frac{r}{R} \cos \theta, \Phi_{c 3}=\frac{r}{R} \sin \theta \\
\Phi_{c(k+3)}=A_{k}\left[J_{n_{k}}\left(\lambda_{k} \frac{r}{R}\right)+C_{k} I_{n_{k}}\left(\lambda_{k} \frac{r}{R}\right)\right] f_{k}(\theta), k=1,2, \ldots
\end{gathered}
$$

where $J_{n_{k}}$ and $I_{n_{k}}$ are the Bessel functions of the first kind and the modified Bessel functions of order $n_{k}$, respectively. The first three modes represent the rigid-body modes and other modes represent the elastic vibration modes. The characteristic values obtained from Eq. (23d) are tabulated in the work of Kwak and Han(2007) by rearranging the values given in reference [Leissa(1993)]. In this case, $\Lambda_{c}$ has the following form.

$$
\Lambda_{c}=\operatorname{diag}\left(\left[\begin{array}{llllllll}
0 & 0 & 0 & \lambda_{1}^{4} & \lambda_{2}^{4} & \lambda_{3}^{4} & \cdots & \lambda_{n_{c}-3}^{4}
\end{array}\right]\right)
$$

\section{Free vibration analysis of rectangular plate with a hole by use of global coordinates}

Let us consider a rectangular plate with a rectangular hole, as shown in Figure 1.

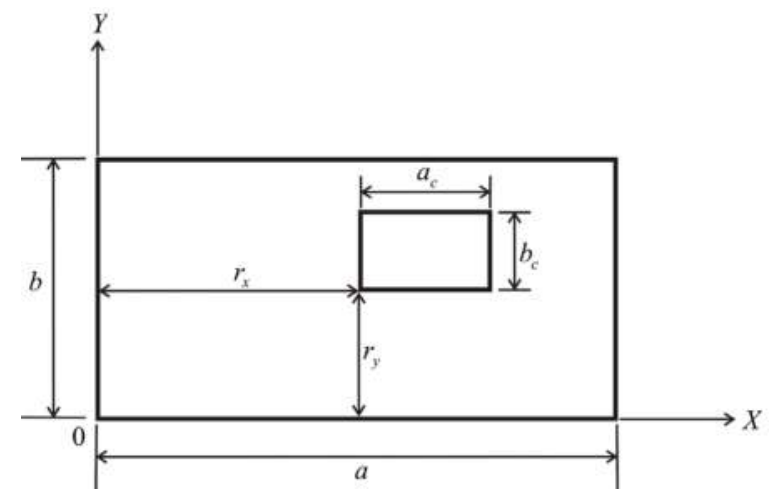

Fig. 1. Rectangular plate with a rectangular hole with global axes.

In this case, the total kinetic and potential energies can be obtained by subtracting the energies belonging to the hole domain from the total energies for the global domain.

$$
\begin{aligned}
& T_{\text {total }}=T_{R}-T_{R H}=\frac{1}{2} \dot{q}_{r}^{T}\left(M_{r}-M_{r h}^{*}\right) \dot{q}_{r}=\frac{1}{2} \dot{q}_{r}^{T} M_{r r h} \dot{q}_{r} \\
& V_{\text {total }}=V_{R}-V_{R H}=\frac{1}{2} q_{r}^{T}\left(K_{r}-K_{r h}^{*}\right) q_{r}=\frac{1}{2} q_{r}^{T} K_{r r h} q_{r}
\end{aligned}
$$

where

$$
M_{r r h}=M_{r}-M_{r h}^{*}, K_{r r h}=K_{r}-K_{r h}^{*}
$$

in which $M_{r}, K_{r}$ are mass and stiffness matrices for the whole rectangular plate, which are given by Eq. (5), and $M_{r h}^{*}, K_{r h}^{*}$ reflect the reductions in mass and stiffness matrices due to 
the hole, which can be also expressed by non-dimensionalized mass and stiffness matrices, respectively.

$$
M_{r h}^{*}=\rho h a b \bar{M}_{r h}^{*}, K_{r h}^{*}=\frac{D b}{a^{3}} \bar{K}_{r h}^{*}
$$

where

$$
\begin{aligned}
& \bar{M}_{r h}^{*}=\int_{\bar{r}_{x}}^{\bar{r}_{x}+\bar{a}_{c}} \int_{\bar{r}_{y}}^{\bar{r}_{y}+\bar{b}_{c}} \Phi_{r}^{T} \Phi_{r} d \xi d \eta \\
& \bar{K}_{r h}^{*}=\int_{\bar{r}_{x}}^{\bar{r}_{x}+\bar{a}_{c}} \int_{\bar{r}_{y}}^{\bar{r}_{y}+\bar{b}_{c}}\left[\frac{\partial^{2} \Phi_{r}^{T}}{\partial \xi^{2}} \frac{\partial^{2} \Phi_{r}}{\partial \xi^{2}}+\alpha^{4} \frac{\partial^{2} \Phi_{r}^{T}}{\partial \eta^{2}} \frac{\partial^{2} \Phi_{r}}{\partial \eta^{2}}+v \alpha^{2}\left(\frac{\partial^{2} \Phi_{r}^{T}}{\partial \xi^{2}} \frac{\partial^{2} \Phi_{r}}{\partial \eta^{2}}+\frac{\partial^{2} \Phi_{r}^{T}}{\partial \eta^{2}} \frac{\partial^{2} \Phi_{r}}{\partial \xi^{2}}\right)\right. \\
& \left.+2(1-v) \alpha^{2} \frac{\partial^{2} \Phi_{r}^{T}}{\partial \xi \partial \eta} \frac{\partial^{2} \Phi_{r}}{\partial \xi \partial \eta}\right] d \xi d \eta
\end{aligned}
$$

in which $\bar{r}_{x}=r_{x} / a, \bar{r}_{y}=r_{y} / b, \bar{a}_{c}=a_{c} / a, \bar{b}_{c}=b_{c} / b$ represent various aspect ratios. Hence, the non-dimensionalized eigenvalue problem for the addressed problem can be expressed as:

$$
\left(\bar{K}_{r r h}-\bar{\omega}^{2} \bar{M}_{r r h}\right) A=0
$$

where

$$
\bar{M}_{r r h}=\bar{M}_{r}-\bar{M}_{r h}^{*}, \bar{K}_{r r h}=\bar{K}_{r}-\bar{K}_{r h}^{*}
$$

To calculate the non-dimensionalized mass and stiffness matrices for the hole domain given by Eq. (28), we generally resort to numerical integration. However, in the case of a simplysupported rectangular plate with a rectangular hole, the exact expressions exists for the nondimensionalized mass and stiffness matrices for the hole[Kwak \& Han(2007)].

\section{Independent coordinate coupling method for a rectangular plate with a rectangular hole}

Let us consider again the rectangular plate with a rectangular hole, as shown in Fig. 2. As can be seen from Fig. 2, the local coordinates fixed to the hole domain is introduced. Considering the non-dimensionalized coordinates, $\xi_{h}=x_{h} / a_{c}, \eta_{h}=y_{h} / b_{c}$, we can express the displacement inside the hole domain as

$$
w_{h}\left(\xi_{h}, \eta_{h}\right)=\Phi_{h}\left(\xi_{h}, \eta_{h}\right) q_{h}
$$

where $\Phi_{h}\left(\xi_{h}, \eta_{h}\right)=\left[\Phi_{h 1} \Phi_{h 2} \ldots \Phi_{h m_{h}}\right]$ is the $1 \times m_{h}$ admissible function matrix, and $q_{h}(t)=\left[q_{h 1} q_{h 2} \ldots q_{h m_{h}}\right]^{T}$ is the $m_{h} \times 1$ generalized coordinate vector. If we apply the separation of variables to the admissible function as we did in Eq. (10), then we have

$$
\Phi_{h i}\left(\xi_{h}, \eta_{h}\right)=\phi_{h i}\left(\xi_{h}\right) \psi_{h i}\left(\eta_{h}\right), i=1,2, \ldots, m_{h}
$$




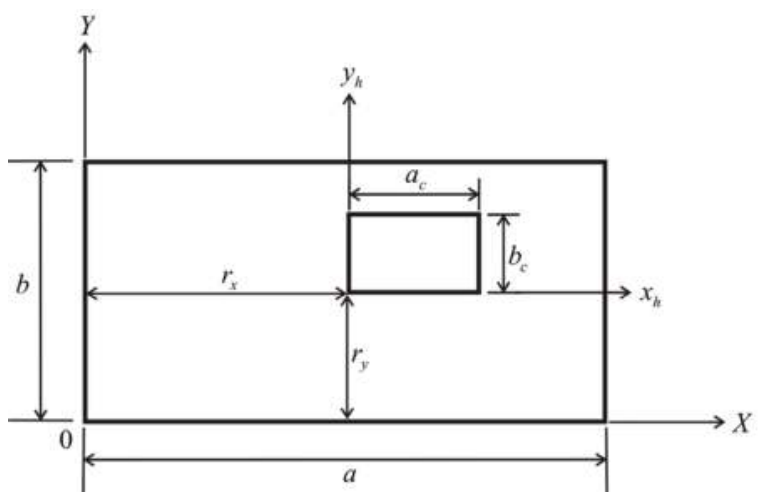

Fig. 2. Rectangular plate with a rectangular hole with local axes.

Using Eqs. (31) and (32), we can express the kinetic and potential energies in the hole domain as

$$
T_{R H}=\frac{1}{2} \dot{q}_{r h}^{T} M_{r h} \dot{q}_{r h}, V_{R H}=\frac{1}{2} q_{r h}^{T} K_{r h} q_{r h}
$$

Hence, the total kinetic and potential energies can be written as

$$
T_{\text {total }}=\frac{1}{2} \dot{q}_{r}^{T} M_{r} \dot{q}_{r}-\frac{1}{2} \dot{q}_{r h}^{T} M_{r h} \dot{q}_{r h}, \quad V_{\text {total }}=\frac{1}{2} q_{r}^{T} K_{r} q_{r}-\frac{1}{2} q_{r h}^{T} K_{r h} q_{r h}
$$

Where $M_{r}, K_{r}$ are defined by Eqs. (5) and (6), and

$$
M_{r h}=\rho h a_{c} b_{c} \bar{M}_{r h}, K_{r h}=\frac{D b_{c}}{a_{c}^{3}} \bar{K}_{r h}
$$

in which

$$
\begin{gathered}
\bar{M}_{r h}=\int_{0}^{1} \int_{0}^{1} \Phi_{h}^{T} \Phi_{h} d \xi_{h} d \eta_{h} \\
\bar{K}_{r h}=\int_{0}^{1} \int_{0}^{1}\left[\frac{\partial^{2} \Phi_{h}^{T}}{\partial \xi_{h}^{2}} \frac{\partial^{2} \Phi_{h}}{\partial \xi_{h}^{2}}+\alpha_{c}^{4} \frac{\partial^{2} \Phi_{h}^{T}}{\partial \eta_{h}^{2}} \frac{\partial^{2} \Phi_{h}}{\partial \eta_{h}^{2}}+v \alpha_{c}^{2}\left(\frac{\partial^{2} \Phi_{h}^{T}}{\partial \xi_{h}^{2}} \frac{\partial^{2} \Phi_{h}}{\partial \eta_{h}^{2}}+\frac{\partial^{2} \Phi_{h}^{T}}{\partial \eta_{h}^{2}} \frac{\partial^{2} \Phi_{h}}{\partial \xi_{h}^{2}}\right)\right. \\
\left.+2(1-v) \alpha_{c}^{2} \frac{\partial^{2} \Phi_{h}^{T}}{\partial \xi_{h} \partial \eta_{h}} \frac{\partial^{2} \Phi_{h}}{\partial \xi_{h} \partial \eta_{h}}\right] d \xi_{h} d \eta_{h}
\end{gathered}
$$

and $\alpha_{c}=a_{c} / b_{c}$. Note that the definite integrals in Eq. (36) has distinctive advantage compared to Eq. (28) because it has an integral limit from 0 to 1 thus permitting closed form expressions. Therefore, we can use the same expression used for the free-edge rectangular plate.

Since the local coordinate system is used for the hole domain, we do not have to carry out integration for the hole domain, as in Eq. (28). However, the displacement matching condition between the global and local coordinates should be satisfied inside the hole domain. The displacement matching condition inside the hole domain can be written as 


$$
w_{r h}\left(\xi_{h}, \eta_{h}\right)=w_{r}(\xi, \eta)
$$

The relationship between the non-dimensionalized global and local coordinates can be written as

$$
\xi=\frac{r_{x}}{a}+\frac{a_{c}}{a} \xi_{h}, \quad \eta=\frac{r_{y}}{b}+\frac{b_{c}}{b} \eta_{h}
$$

Considering Eqs. (3), (10), (31) and (32), and inserting them into Eq. (37), we can derive

$$
\sum_{j=1}^{m_{h}} \Phi_{r h j}\left(\xi_{h}, \eta_{h}\right) q_{r h j}(t)=\sum_{j=1}^{m_{h}} \phi_{h j}\left(\xi_{h}\right) \psi_{h j}\left(\eta_{h}\right) q_{r h j}(t)=\sum_{k=1}^{m} \Phi_{r k}(\xi, \eta) q_{r k}(t)=\sum_{k=1}^{m} \phi_{k}(\xi) \psi_{k}(\eta) q_{r k}(t)
$$

Multiplying Eq. (39) by $\phi_{h i}\left(\xi_{h}\right) \psi_{h i}\left(\eta_{h}\right)$ and performing integration, we can derive

$$
\begin{aligned}
& \sum_{j=1}^{m_{h}} \int_{0}^{1} \int_{0}^{1} \phi_{h i}\left(\xi_{h}\right) \psi_{h i}\left(\eta_{h}\right) \phi_{h j}\left(\xi_{h}\right) \psi_{h j}\left(\eta_{h}\right) d \xi_{h} d \eta_{h} q_{r h j}(t)= \\
& \quad=\sum_{k=1}^{m} \int_{0}^{1} \int_{0}^{1} \phi_{h i}\left(\xi_{h}\right) \psi_{h i}\left(\eta_{h}\right) \phi_{r k}(\xi) \psi_{r k}(\eta) d \xi_{h} d \eta_{h} q_{r k}(t), \quad i=1,2, \ldots, m_{h}
\end{aligned}
$$

Using the orthogonal property of the eigenfunctions of the uniform beam, Eq. (40) can be rewritten as

$$
\begin{aligned}
q_{r h i}(t) & =\sum_{k=1}^{m} \int_{0}^{1} \phi_{h i}\left(\xi_{h}\right) \phi_{k}(\xi) d \xi_{h} \int_{0}^{1} \psi_{h i}\left(\eta_{h}\right) \psi_{k}(\eta) d \eta_{h} q_{r k}(t) \\
& =\sum_{k=1}^{m}\left(T_{r r h}\right)_{i k} q_{r k}(t), i=1,2, \ldots, m_{h}
\end{aligned}
$$

If we express Eq. (41) in the matrix form, we can have

$$
q_{r h}=T_{r r h} q_{r}
$$

where $T_{r r h}$ is the $m_{h} \times m$ transformation matrix between two coordinates. Inserting Eq. (42) into Eq. (34), we can derive

$$
\begin{aligned}
& T_{\text {total }}=\frac{1}{2} \dot{q}_{r}^{T} M_{r} \dot{q}_{r}-\frac{1}{2} \dot{q}_{r h}^{T} T_{r r h}^{T} M_{r h} T_{r r h} \dot{q}_{r h}=\frac{1}{2} \dot{q}_{r} M_{r r h} \dot{q}_{r} \\
& V_{\text {total }}=\frac{1}{2} q_{r}^{T} K_{r} q_{r}-\frac{1}{2} q_{r h}^{T} T_{r r h}^{T} K_{r h} T_{r r h} q_{r h}=\frac{1}{2} q_{r}^{T} K_{r r h} q_{r}
\end{aligned}
$$

where

$$
M_{r r h}=M_{r}-T_{r r h}^{T} M_{r h} T_{r r h}, K_{r r h}=K_{r}-T_{r r h}^{T} K_{r h} T_{r r h}
$$

Equation (44) can be expressed by means of non-dimensionalized parameters

$$
M_{r r h}=\rho h a b \bar{M}_{r r h}, K_{r r h}=\frac{D b}{a^{3}} \bar{K}_{r r h}
$$


where

$$
\bar{M}_{r r h}=\bar{M}_{r}-\left(\bar{a}_{c} \bar{b}_{c}\right) T_{r r h}^{T} \bar{M}_{r h} T_{r r h}, \bar{K}_{r r h}=\bar{K}_{r}-\frac{\bar{b}_{c}}{\bar{a}_{c}^{3}} T_{r r h}^{T} \bar{K}_{r h} T_{r r h}
$$

Hence, the non-dimensionalized eigenvalue problem can be written in the same form as Eq. (29).

In deriving the mass and stiffness matrices, Eq. (46), for the eigenvalue problem, we only needed the transformation matrix, $T_{r r h} . \bar{M}_{r}, \bar{K}_{r}$ can be easily computed by Eq. (11) according to the edge boundary conditions and $\bar{M}_{r h}, \bar{K}_{r h}$ can be computed from the results of Eq. (11) for the all free-edge rectangular plate. On the other hand, the computation of $\bar{M}_{r h}^{*}, \bar{K}_{r h}^{*}$ based on the global coordinates is not easy because of integral limits. Compared to the approach based on the global coordinates, the numerical integration for the transformation matrix, $T_{r r h}$, is easy because the integral limits are 0 and 1 . The process represented by Eqs. (42) and (46) is referred to as the ICCM in the study by Kwak and Han(2007). The ICCM enables us to solve the free vibration problem of the rectangular plate with a rectangular hole more easily than the previous approaches based on the global coordinates do. The advantage of the ICCM becomes more apparent when we deal with a circular hole, as will be demonstrated in the next section.

\section{Free vibration analysis of rectangular plate with multiple rectangular cutouts by independent coordinate coupling method}

As in the case of single rectangular hole, the total energy can be computed by subtracting the energy belonging to holes from the energy of the whole rectangular plate, which is not an easy task when applying the classical Rayleigh-Ritz method. However, the ICCM enables us to formulate the free vibration problem for the rectangular plate with multiple holes more easily than the CRRM.

Let us consider a rectangular plate with $n$ rectangular holes as shown in Fig. 3.

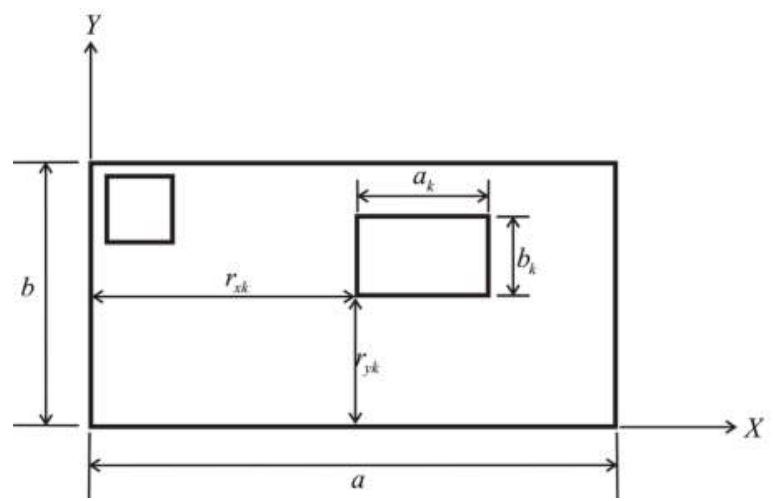

Fig. 3. Rectangular plate with multiple rectangular holes

By employing the same formulation used in the case of a rectangular hole with a single rectangular hole, the non-dimensionalized mass and stiffness matrices can be derived considering a single hole case: 


$$
\bar{M}_{r r h}=\bar{M}_{r}-\sum_{k=1}^{n}\left(\bar{a}_{k} \bar{b}_{k}\right) T_{r r h k}^{T} \bar{M}_{r h} T_{r r h k}, \bar{K}_{r r h}=\bar{K}_{r}-\sum_{k=1}^{n} \frac{\bar{b}_{k}}{\bar{a}_{k}^{3}} T_{r r h k}^{T} \bar{K}_{r h} T_{r r h k}
$$

where the following non-dimensionalized variables are introduced for the analysis

$$
\bar{r}_{x k}=r_{x k} / a, \bar{r}_{y k}=r_{y k} / b, \bar{a}_{k}=a_{k} / a, \bar{b}_{k}=b_{k} / b
$$

And the transformation matrix can be expressed by considering Eq. (41)

$$
\left(T_{r r h k}\right)_{i j}=\int_{0}^{1} \phi_{h i}\left(\xi_{h i}\right) \phi_{j}(\xi) d \xi_{h i} \int_{0}^{1} \psi_{h i}\left(\eta_{h i}\right) \psi_{j}(\eta) d \eta_{h i}
$$

In order to validate the efficacy of the ICCM for the rectangular plate with multiple rectangular holes, the rectangular plate with two square holes as shown in Fig. 4 is considered as a numerical example, in which $v=0.3$. The results of the ICCM are compared to those obtained by the classical Rayleigh-Ritz method.

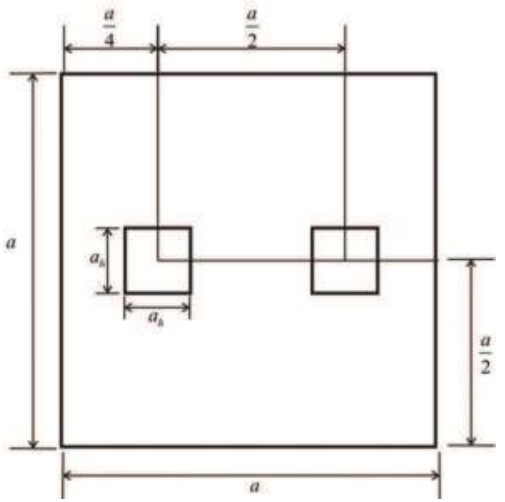

Fig. 4. Square plate with two square holes

Ten admissible functions in each direction were employed, which implies one hundred admissible functions, for both CRRM and ICCM. In the case of the ICCM, the additional admissible functions are necessary for the hole domain. In our study ten admissible functions in each direction of the rectangular hole domain, which implies one hundred admissible functions, were used. The number of admissible functions guaranteeing the convergence are referred to the work of Kwak and Han(2007).

Fig. 5 shows the non-dimensionalized natural frequencies obtained by the CRRM and ICCM for the case that the plate shown in Fig. 4 has all simply-supported boundary conditions, where $\bar{a}_{h}=a_{h} / a$. As shown in Fig. 5 , the results obtained by the ICCM agree well with the results obtained by the CRRM. The fundamental frequency increases as the size of the hole increases but higher natural frequencies undergo rapid change as the size of the hole increases. This result is similar to the one obtained by Kwak and Han(2007) for a single hole case.

In the case of the simply-supported rectangular plate with a hole, the solutions of integrals can be obtained in a closed form without numerical integral technique. However, in the case of the clamped rectangular plate, the closed-form solution can't be obtained, so the 
numerical integrations are necessary. Figure 6 shows the advantage of the ICCM over the CRRM regarding the computational time. As can be seen from Fig. 6, the computational time increases enormously in the case of the CRRM compared to the ICCM as the size of the hole increases. Hence, it can be readily recognized that the ICCM has the computational efficiency compared to the CRRM, which was confirmed in the work of Kwak and Han(2007) for a single hole case.

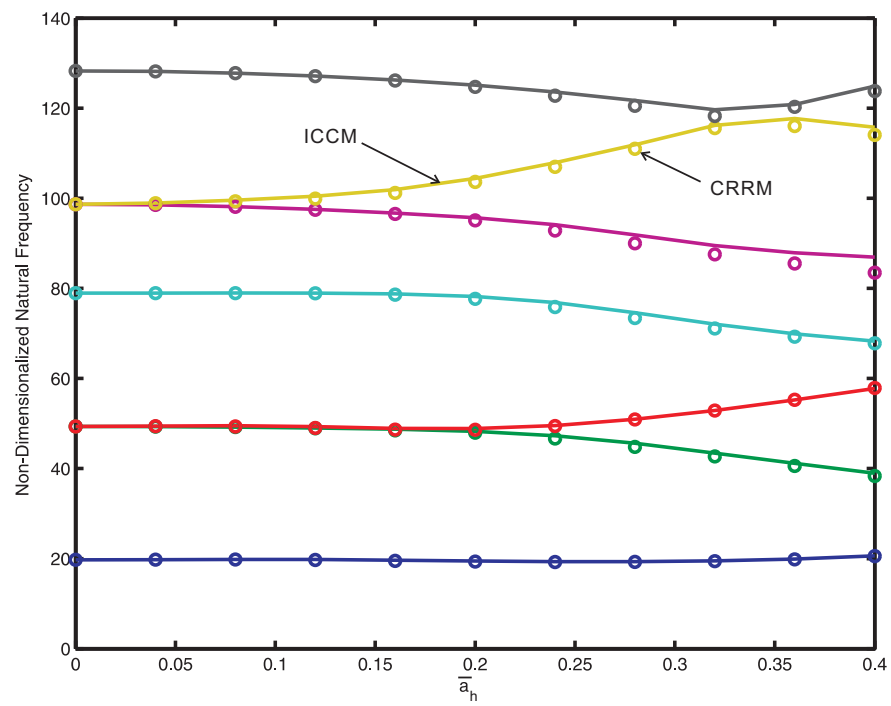

Fig. 5. Simply-supported square plate with two square holes

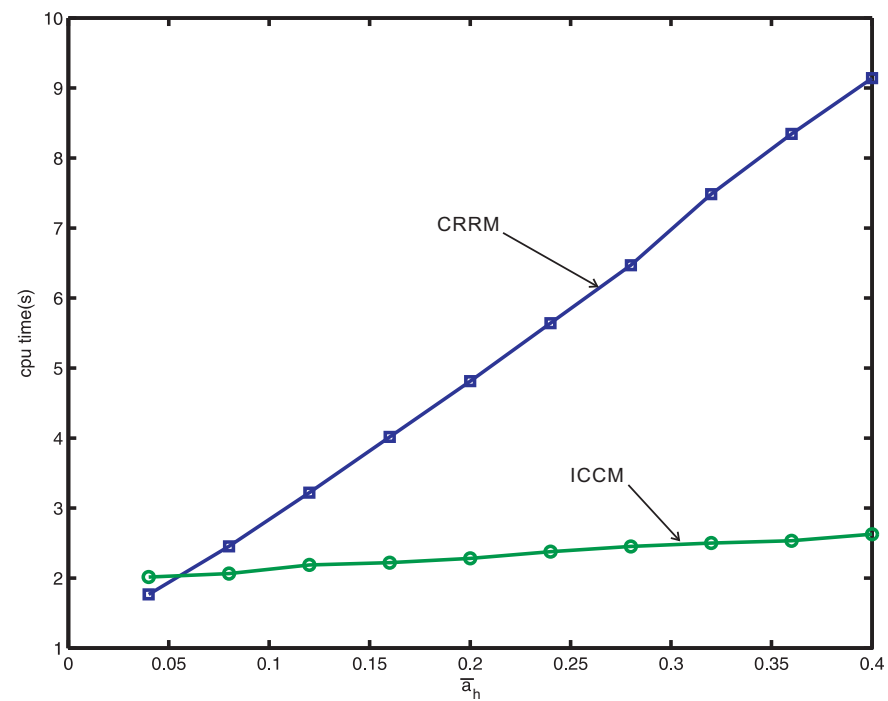

Fig. 6. CPU time vs. hole size 


\section{Independent coordinate coupling method for a rectangular plate with a circular hole}

Let us consider a rectangular plate with a circular hole, as shown in Fig. 7. The global coordinate approach used in Section 4 can be used for this problem but we must resort to numerical integration technique. If we use the ICCM, we can avoid the complex numerical computation and thus simplify the computation as in the case of a rectangular hole.

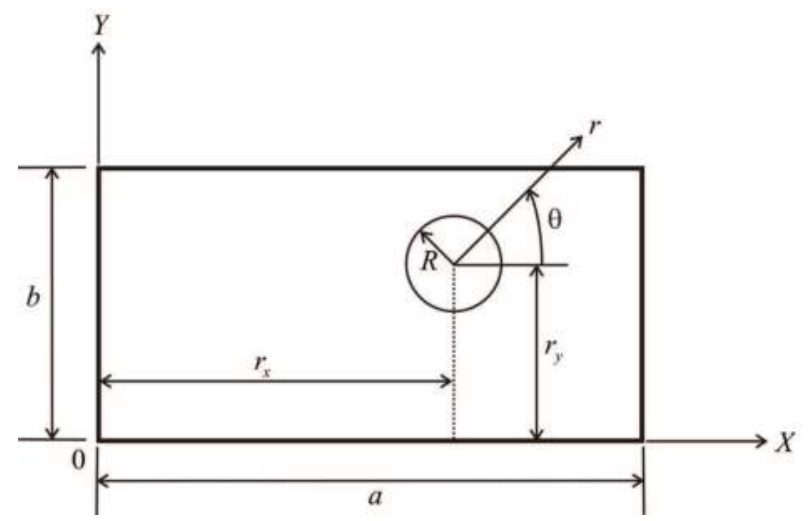

Fig. 7. Rectangular plate with a circular hole.

The total kinetic and potential energies of the rectangular plate with a circular hole are obtained by subtracting the energies of the circular hole domain from the energies of the whole plate, as we did for the case of a rectangular hole. Hence, the following equations can be obtained by using Eqs. (4) and (21).

$$
T_{\text {total }}=\frac{1}{2} \dot{q}_{r}^{T} M_{r} \dot{q}_{r}-\frac{1}{2} \dot{q}_{c h}^{T} M_{c h} \dot{q}_{c h}, \quad V_{\text {total }}=\frac{1}{2} q_{r}^{T} K_{r} q_{r}-\frac{1}{2} q_{c h}^{T} K_{c h} q_{c h}
$$

In order to apply the ICCM, the displacement matching condition should be satisfied. Hence, the following condition should be satisfied inside the circular hole domain.

$$
w_{c}(r, \theta)=w_{r}(\xi, \eta)
$$

Considering Eqs. (20), (3) and (10), we can obtain.

$$
\sum_{j=1}^{m_{c}} \Phi_{c j}(r, \theta) q_{c h j}(t)=\sum_{k=1}^{m} \Phi_{r k}(\xi, \eta) q_{r k}(t)=\sum_{k=1}^{m} \phi_{k}(\xi) \psi_{k}(\eta) q_{r k}(t)
$$

Multiplying Eq. (52) by $\Phi_{c i}(r, \theta)$ and performing integration over the circular hole domain result in

$$
\begin{gathered}
\sum_{j=1}^{m_{c}} \int_{0}^{2 \pi} \int_{0}^{R} \Phi_{c i}(r, \theta) \Phi_{c j}(r, \theta) r d r d \theta q_{c h j}(t)=\sum_{k=1}^{m} \int_{0}^{2 \pi} \int_{0}^{R} \Phi_{c i}(r, \theta) \phi_{k}(\xi) \psi_{k}(\eta) r d r d \theta q_{r k}(t), \\
i=1,2, \ldots, m_{c}
\end{gathered}
$$


Using the orthogonal property of $\Phi_{c i}(r, \theta)$, Eq. (53) can be rewritten as

$$
q_{c h i}(t)=\sum_{k=1}^{m} \int_{0}^{2 \pi} \int_{0}^{R} \Phi_{c i}(r, \theta) \phi_{k}(\xi) \psi_{k}(\eta) r d r d \theta q_{r k}(t)=\sum_{k=1}^{m}\left(T_{c h}\right)_{i k} q_{r k}(t), i=1,2, \ldots, m_{c}
$$

Equation (54) can be expressed in matrix form.

$$
q_{c h}=T_{r c h} q_{r}
$$

where $T_{c h}$ is a $m_{c} \times m$ transformation matrix. We also need the relationship between the global and local coordinates, which can be expressed as follows.

$$
\xi=\frac{r_{x}}{a}+\frac{r \cos \theta}{a}, \quad \eta=\frac{r_{y}}{b}+\frac{r \sin \theta}{b}
$$

Using Eq. (55), the mass and stiffness matrices can be easily derived as in the case of a rectangular hole.

$$
M_{r c h}=M_{r}-T_{r c h}^{T} M_{c h} T_{r c h}, K_{r c h}=K_{r}-T_{r c h}^{T} K_{c h} T_{r c h}
$$

Eq. (57) can be nondimensionalized using Eqs. (5) and (22) as for the rectangular hole. Hence, we obtain

$$
M_{r c h}=\rho h a b \bar{M}_{r c h}, K_{r c h}=\frac{D b}{a^{3}} \bar{K}_{r c h}
$$

where

$$
\bar{M}_{r c h}=\bar{M}_{r}-\left(\pi \alpha \beta^{2}\right) T_{r c h}^{T} T_{r c h}, \bar{K}_{r c h}=\bar{K}_{r}-\left(\frac{\pi \alpha}{\beta^{2}}\right) T_{r c h}^{T} \Lambda_{c} T_{r c h}
$$

in which $\beta=R / a$.

As shown in the process from Eq. (55), (57) and (59), it can be readily seen that the application of the ICCM is very straightforward and the theoretical background is solid. The efficacy of the ICCM are fully demonstrated in the numerical results[Heo and Kwak(2008), Kwak et al.(2005,2006,2007)].

\section{Free vibration analysis of rectangular plate with multiple circular cutouts by independent coordinate coupling method}

Let us consider a rectangular plate with multiple circular holes as shown in Fig. 8. We can easily extend the formulation developed in the previous section to the case of a rectangular plate with multiple circular holes. The resulting mass and stiffness matrices can be expressed as:

$$
\bar{M}_{r}=\bar{M}-\sum_{k=1}^{n} \pi \alpha \beta_{k}^{2} T_{r c h k}^{T} T_{r c h k}, \bar{K}_{r c h}=\bar{K}_{r}-\sum_{k=1}^{n} \frac{\pi \alpha}{\beta_{k}^{2}} T_{r c h k}^{T} \Lambda_{c} T_{r c h k}
$$


where $T_{\text {rchk }}$ represents the transformation matrix for $k$ th circular hole

$$
\left(T_{r c h k}\right)_{i j}=\int_{0}^{2 \pi} \int_{0}^{R_{k}} \Psi_{k i}\left(r_{k}, \theta_{k}\right) \phi_{j}(\xi) \psi_{j}(\eta) r d r d \theta
$$

We also need the relationship between the global and local coordinates, which can be expressed as follows:

$$
\xi=\frac{r_{k x}}{a}+\frac{r_{k} \cos \theta_{k}}{a}, \eta=\frac{r_{k y}}{b}+\frac{r_{k} \sin \theta_{k}}{b}
$$

For the numerical study, we considered a square plate with two circular holes as shown in Fig. 9. The results of the ICCM were compared to those obtained by the commercial finite element method, ANSYS. $v=0.3, E=76 \mathrm{GPa}, a=1 \mathrm{~m}, \rho=7800 \mathrm{~kg} / \mathrm{m}^{3}$ were used and nondimensionalized frequencies were estimated from the computed natural frequencies. For the ICCM, ten admissible functions were used for each direction of the square plate and fifty admissible functions were used for each circular hole.

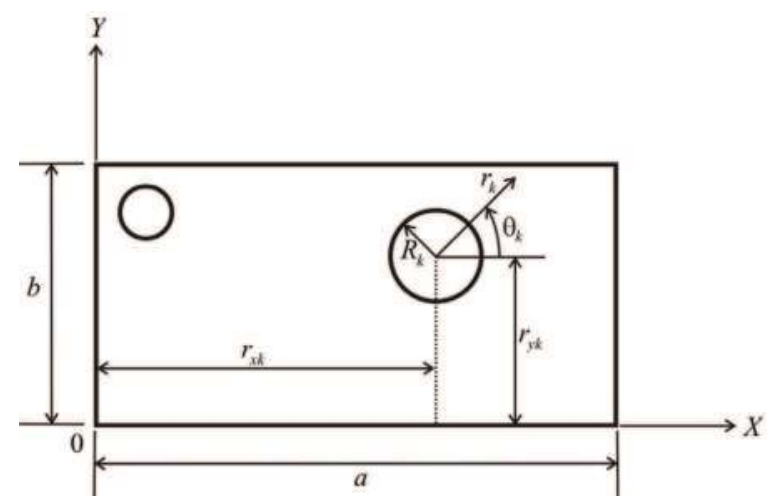

Fig. 8. Rectangular plate with multiple circular holes with local axes

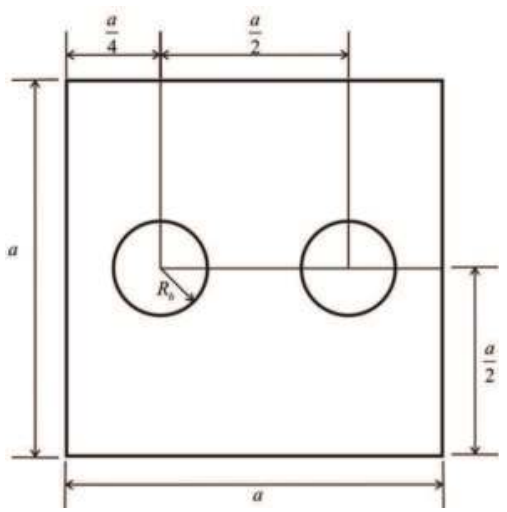

Fig. 9. Square plate with two circular holes 
Figure 10 shows the non-dimensionalized natural frequencies obtained by the ICCM and ANSYS for simply-supported square plate with two circular hole, where $\beta=R_{h} / a$. As shown in the figure, the results obtained by the ICCM are in good agreement with those obtained by ANSYS.

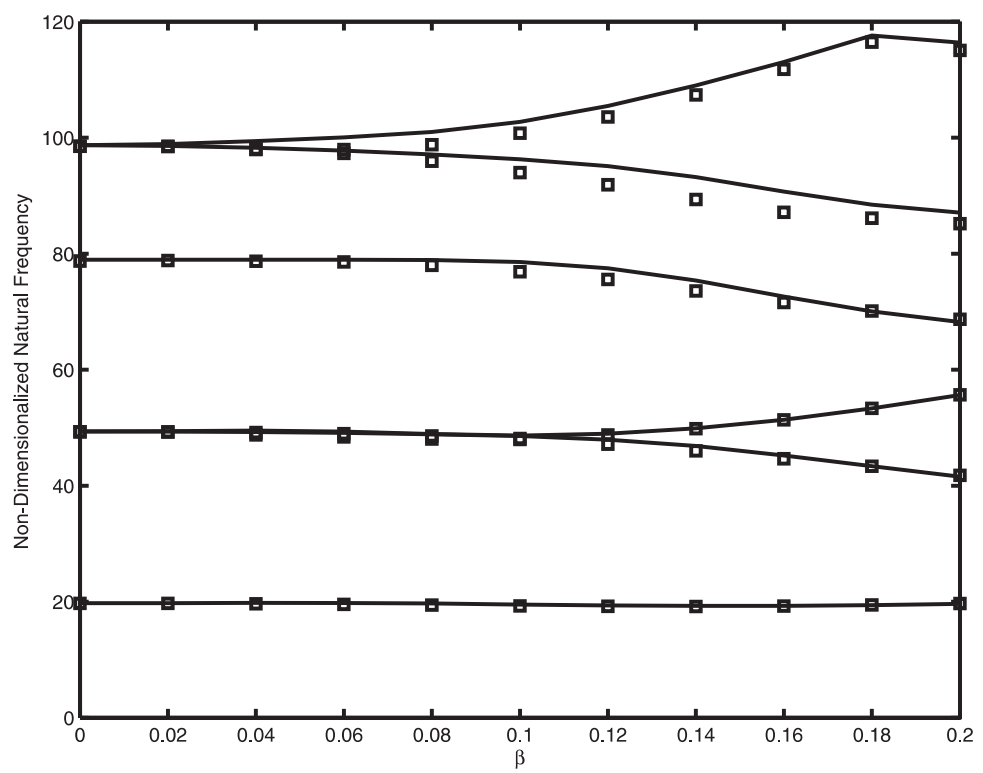

Fig. 10. Simply-supported square plate with two circular holes ( -: ICCM, $\square$ :ANSYS)

\section{Independent coordinate coupling method for a circular plate with an eccentric circular hole}

Let us consider a circular plate with an eccentric circular hole as shown in Fig. 11 to demonstrate the efficacy of the ICCM.

The total kinetic and potential energies can be written as

$$
T_{\text {total }}=T_{C}-T_{C H}, V_{\text {total }}=V_{C}-V_{C H} .
$$

However, it is not easy to express the energies belonging to the eccentric circular hole using the global coordinate system whose origin is fixed to the circular plate since the integral limits cannot be easily established. In addition, the numerical integration for the eccentric circular hole is also not an easy task. These complexities can be avoided with the use of the ICCM [Heo and Kwak(2008)]. Based on the ICCM, the deflection of the circular plate with the eccentric circular hole can be expressed as a combination of eigenfunctions and generalized coordinates, which are based on the local coordinates, $r_{c}, \theta_{c}$, as shown in Fig.11. Inserting Eq. (21) into Eq. (63), the total kinetic and potential energies can be written as

$$
T_{\text {total }}=\frac{1}{2} \dot{q}_{c}^{T} M_{c} \dot{q}_{c}-\frac{1}{2} \dot{q}_{c h}^{T} M_{c h} \dot{q}_{c h}, V_{\text {total }}=\frac{1}{2} q_{c}^{T} K_{c} q_{c}-\frac{1}{2} q_{c h}^{T} K_{c h} q_{c h}
$$




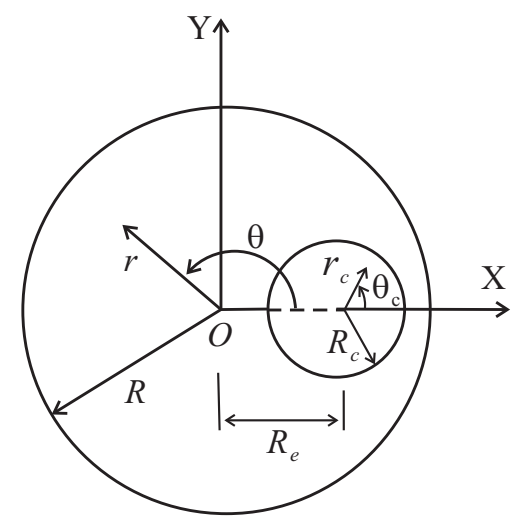

Fig. 11. Circular plate with an eccentric hole for coordinate system

In the next task in the ICCM, the displacement matching condition is satisfied inside the eccentric circular hole domain, i.e.

$$
w_{c h}\left(r_{c}, \theta_{c}\right)=w_{c}(r, \theta) .
$$

Inserting Eqs. (20) into (65), we then obtain

$$
\sum_{j=1}^{n_{c}} \Phi_{c j}\left(r_{c}, \theta_{c}\right) q_{c h j}(t)=\sum_{j=1}^{n} \Phi_{j}(r, \theta) q_{c j}(t)
$$

Multiplying Eq. (661) by $\Phi_{c i}\left(r_{c}, \theta_{c}\right)$ and integrating over the eccentric circular hole domain result in

$$
\begin{gathered}
\sum_{j=1}^{n_{c}} \int_{0}^{2 \pi} \int_{0}^{R_{c}} \Phi_{c i}\left(r_{c}, \theta_{c}\right) \Phi_{c j}\left(r_{c}, \theta_{c}\right) r_{c} d r_{c} d \theta_{c} q_{c h j}(t)=\sum_{j=1}^{n} \int_{0}^{2 \pi} \int_{0}^{R_{c}} \Phi_{c i}\left(r_{c}, \theta_{c}\right) \Phi_{j}(r, \theta) r_{c} d r_{c} d \theta_{c} q_{j}(t) . \\
i=1,2, \ldots, n_{c}
\end{gathered}
$$

Using the orthogonal property of $\Phi_{c i}\left(r_{c}, \theta_{c}\right)$, Eq. (67) can be rewritten as

$$
q_{c h i}(t)=\sum_{j=1}^{n} \int_{0}^{2 \pi} \int_{0}^{R_{c}} \Phi_{c i}\left(r_{c}, \theta_{c}\right) \Phi_{j}(r, \theta) r_{c} d r_{c} d \theta_{c} q_{j}(t)=\sum_{k=1}^{n}\left(T_{c c h}\right)_{i k} q_{j k}(t), i=1,2, \ldots, n_{c} .
$$

Equation (68) can be expressed in matrix form

$$
q_{c h}=T_{c c h} q_{c}
$$

where $T_{c c h}$ is a $n_{c} \times n$ transformation matrix. The relationships between the global and local coordinates are needed to compute each element in the transformation matrix, which can be expressed as follows.

$$
r=\sqrt{r_{c}^{2}+R_{e}^{2}-2 r_{c} R_{e} \cos \left(\pi-\theta_{c}\right)}, \quad \theta=\tan ^{-1}\left(\frac{r_{c} \sin \left(\pi-\theta_{c}\right)}{R_{e}+r_{c} \cos \theta_{c}}\right) .
$$


Using Eqs. (69) into (64), we can derive the mass and stiffness matrices as follows:

$$
M_{c c h}=M_{c}-T_{c c h}^{T} M_{c h} T_{c c h}, K_{c c h}=K_{c}-T_{c c h}^{T} K_{c h} T_{c c h} .
$$

which can be expressed in terms of the non-dimensionalized mass and stiffness matrices

$$
M_{c c h}=\rho h \pi R^{2} \bar{M}_{c c h}, K_{c c h}=\frac{D \pi}{R^{2}} \bar{K}_{c c h}
$$

where

$$
\bar{M}_{c c h}=I-\alpha^{2} T_{c c h}^{T} T_{c c h}, \bar{K}_{c c h}=\Lambda-\frac{1}{\alpha^{2}} T_{c c h}^{T} \Lambda_{c} T_{c c h}
$$

in which $\alpha=R_{c} / R$ is the ratio of the radius of the eccentric circular hole to the radius of the circular plate. Hence, the non-dimensionalized eigenvalue problem for the circular plate with an eccentric circular hole can be expressed as

$$
\left[\bar{K}_{c c h}-\bar{\omega}^{2} \bar{M}_{c c h}\right] A=0
$$

where $\bar{\omega}=\omega \sqrt{\rho h R^{4} / D}$ is the non-dimensionalized natural frequency.

The finite element commercial code, ANSYS, was used for the calculation of nondimensionalized natural frequencies of the simply-supported circular plate with an eccentric circular hole, where material constants, $\rho=2700 \mathrm{~kg} / \mathrm{m}^{3}, E=69 G P a, v=0.3$ and $h=2 \mathrm{~mm}$, $R=1 \mathrm{~m}$ were used. Figure 12 shows the mesh configuration of two cases for $\alpha=0.25, e=0.4$ and $\alpha=0.5, e=0.4$, respectively, where the non-dimensionalized eccentric constant, $e=R_{e} / R$, is introduced. The mesh for the first case consisted of 4261 elements and 4395 nodes and the mesh for the second case consisted of 3197 elements and 3357 nodes.

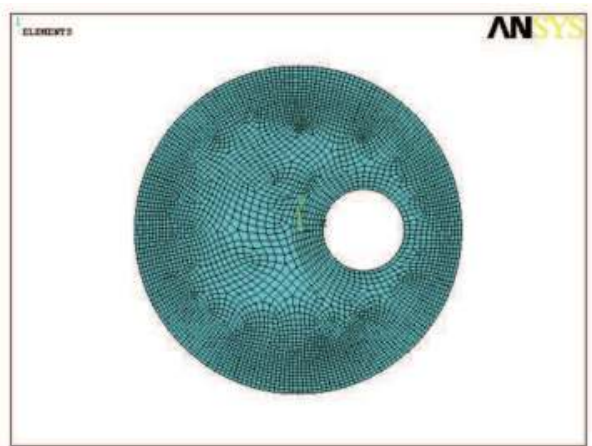

(a) $\alpha=0.25, e=0.4$

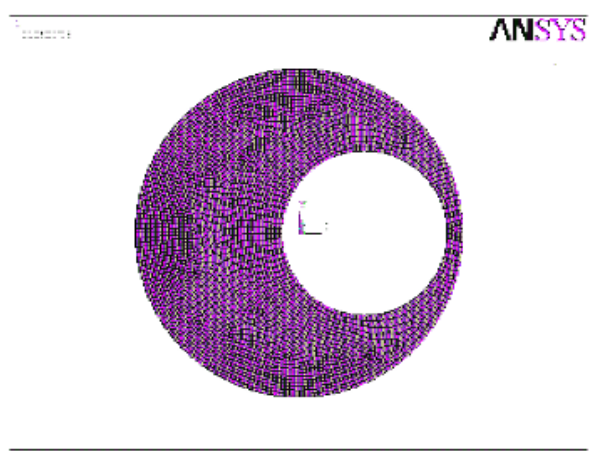

(b) $\alpha=0.5, e=0.4$

Fig. 12. Mesh Configurations by ANSYS

Figures 13 and 14 show the changes in the non-dimensionalized natural frequencies of the simply-supported circular plate with an eccentric circular hole with respect to the eccentricity when $\alpha=0.25$ and 0.5 , respectively. Figs. 13 and 14 show the good agreement between the results obtained by the ICCM and the results by ANSYS. Eccentricity had a small effect on the 
fundamental mode, regardless of the hole size. However, the increases of the hole size and eccentricity had a large effect on higher natural frequencies, which changed unpredictably. Instead of commercial finite element codes, the ICCM can be used as an effective tool for the estimation of natural frequencies of a circular plate with an eccentric circular hole. Different boundary conditions were treated in the work by Heo and Kwak(2008).

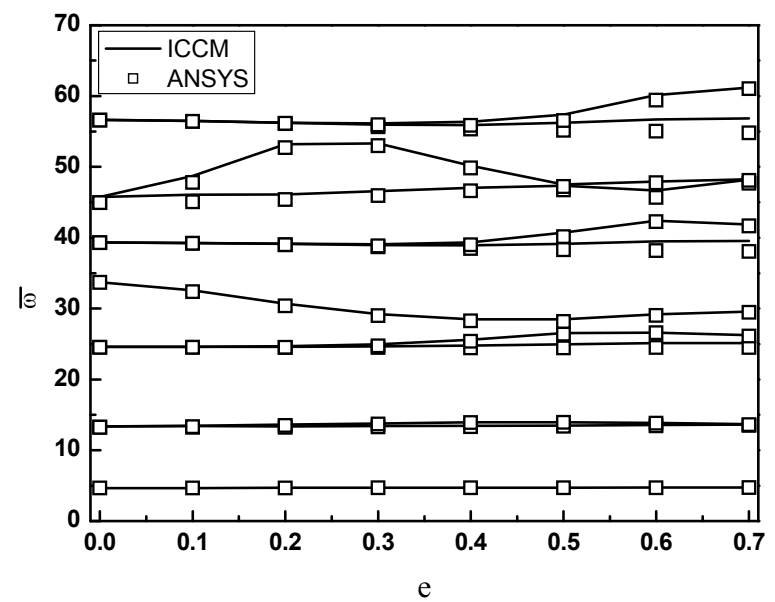

Fig. 13. Non-Dimensionalized Natural Frequency vs. Eccentricity for $\alpha=0.25$

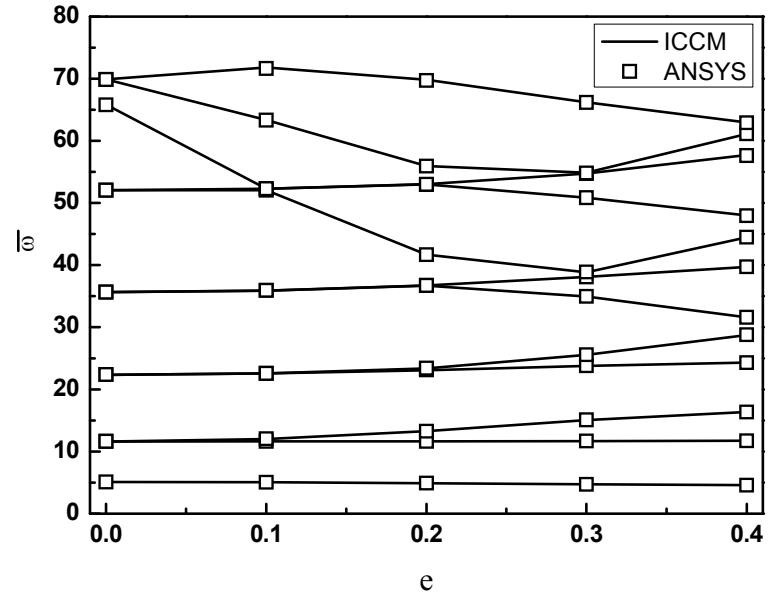

Fig. 14. Non-Dimensionalized Natural Frequency vs. Eccentricity for $\alpha=0.5$

\section{Discussion and conclusions}

In general, the free vibration problem of a plate with holes can't be solved analytically. Therefore, we have to resort to numerical approach such as the finite element method. The 
classical Rayleigh-Ritz method has been popularly used for the analysis of a uniform rectangular plate and the exact solution exists for uniform circular plate. The procedure of the classical Rayleigh-Ritz method was first explained in detail. In applying the classical Rayleigh-Ritz method based on the global coordinates only, the kinetic and potential energies of the rectangular plate with a hole were calculated by subtracting the hole domain in the integrals. However, the Rayleigh-Ritz method can't be effectively used when the plate has holes because the numerical computation of integrals is required. If the plate hole geometry belongs to either rectangular or circular shape, the newly developed method, so called the independent coordinate coupling method (ICCM) can be effectively used. The ICCM has proved its effectiveness in analyzing the free vibration of a rectangular plate with a rectangular hole, a rectangular plate with multiple rectangular holes, a rectangular plate with a circular hole, a rectangular plate with multiple circular holes, and a circular plate with a circular hole. However, the ICCM can be easily extended to a circular plate with a rectangular hole and circular plate with multiple circular holes.

To apply the ICCM to the addressed problem, the global coordinates are set up for the plate and the local coordinates are set up for the hole domain, independently. The kinetic and potential energy expressions for the plate and the inner hole were then derived independently. Since the plate inside the hole domain can be regarded as a virtual free-edge plate, the energies, which are to be subtracted from the total energies, can be easily expressed in closed form. The resulting total energies are expressed in terms of generalized coordinates, which belong to either global or local coordinates. Hence, we need to unify the generalized coordinates. To this end, the relationships between the generalized coordinates belonging to the global and local coordinates were then derived using the displacement matching condition inside the hole domain and the orthogonal property of the admissible functions. In this way, the total kinetic and potential energies can be easily obtained and used for the calculation of the natural frequencies and modes of the circular plate with holes. To verify results of the proposed ICCM, numerical calculations were carried out using the classical Rayleigh-Ritz method based on the global coordinates only and the commercial finite element program. Experiments have been also carried out for the free-edge square plate with a square and circular hole. Both numerical and experimental results showed that good agreement exists between the results by the ICCM and the results obtained by the different algorithms and experiments. Hence, it can be concluded that the proposed ICCM can be effectively used for the free vibration analysis of a plate with holes.

\section{References}

Aksu, G. \& Ali, R. (1976). Determination of Dynamic Characteristics of Rectangular Plates with Cut-outs Using a Finite Difference Formulation. Journal of Sound and Vibration, Vol. 44, (147-158), ISSN0022-460X

Ali, R. \& Atwal, S. J. (1980). Prediction of Natural Frequencies of Vibration of Rectangular Plates with Rectangular Cutouts. Computers and Structures, Vol. 12, No. 9, (819-823), ISSN0045-7949

Avalos, D. R. \& Laura, P. A. A. (2003). Transverse Vibrations of Simply Supported Rectangular Plates with Two Rectangular Cutouts. Journal of Sound and Vibration, Vol.267, (967-977), ISSN0022-460X 
Bhat, R. B. (1985). Natural Frequencies of Rectangular Plates Using Characteristic Orthogonal Polynomials in Rayleigh-Ritz Method. Journal of Sound and Vibration, Vol. 102, (493-499), ISSN0022-460X

Bhat, R. B. (1985). Plate Deflections Using Orthogonal Polynomials. American Society of Civil Engineers, Journal of the Engineering Mechanics Division, Vol. 111, (1301-1309), ISSN0044-7951

Bhat, R. B. (1990). Numerical Experiments on the Determination of Natural Frequencies of Transverse Vibrations of Rectangular Plates of Nonuniform Thickness. Journal of Sound and Vibration, Vol. 138, (205-219), ISSN0022-460X

Cheng, L.; Li, Y. Y. \& Yam, L. H. (2003). Vibration Analysis of Annular-Like Plates. Journal of Sound and Vibration, Vol. 262, (1153-1170), ISSN0022-460X

Eastep, F.E. \& Hemmig, F.G. (1978). Estimation of Fundamental Frequency of Non-Circular Plates with Free, Circular Cutouts. Journal of Sound and Vibration, Vol. 56, No. 2, (155-165), ISSN0022-460X

Hegarty, R.F. \& Ariman, T. (1975). Elasto-Dynamic Analysis of Rectangular Plates with Circular Holes. Int. J. Solids Structures, Vol. 11, (895-906), ISSN0020-7683

Heo, S. \& Kwak, M. K. (2008). Free Vibration Analysis of an Annular Plate by the Independent Coordinate Coupling Method. Transactions of the Korean Society for Noise and Vibration Engineering, Vol.18 No.5, (564-571), ISSN1598-2785

Heo, S \& Kwak, M. K. (2008). Free Vibration Analysis of a Circular Plate with an Eccentric Circular Hole by the Independent Coordinate Coupling Method. Transactions of the Korean Society for Noise and Vibration Engineering, Vol.18 No.6, (681-689), ISSN15982785

Itao, K. \& Crandall, S.H. (1979). Natural Modes and Natural Frequencies of Uniform, Circular, Free-Edge Plates. Journal of Applied Mechanics, Vol. 46, (448-453), ISSN0021-8936

Joga-Rao, C. V. \& Pickett, G. (1961). Vibrations of Plates of Irregular Shapes and Plates with Holes. Journal of the Aeronautical Society of India, Vol. 13, No. 3, (83-88), ISSN00019267

Khurasia, H. B. \& Rawtani, S. (1978). Vibration Analysis of Circular Plates with Eccentric Hole. Journal of Applied Mechanics, Vol.45, No.1, (215 217), ISSN0021-8936

Kim, K.C.; Han, S.Y. \& Jung, J.H. (1987). Transverse Vibration of Stiffened Rectangular Plates Having an Inner Cutout. Journal of the Society of Naval Architects of Korea, Vol. 24, No. 3, (35-42), ISSN1225-1143

Kumai, T. (1952). The Flexural Vibrations of a Square Plate with a Central Circular Hole. Proc. $2^{\text {nd }}$ Japan Natl. Congr. Appl. Mech., (339-342), ISSN0448-8660

Kwak, M. K. \& Han, S. B. (2005). Free Vibration Analysis of Rectangular Plate with a Rectangular Cutout by Independent Coordinate Coupling Method. Transactions of the Korean Society for Noise and Vibration Engineering, Vol. 15, No. 12, (1398-1407), ISSN1598-2785

Kwak, M. K. \& Han, S. B. (2006). Free Vibration Analysis of Simply-supported Rectangular Plate with a Circular Cutout by Independent Coordinate Coupling Method. Transactions of the Korean Society for Noise and Vibration Engineering, Vol. 16, No. 6, (643-650), ISSN1598-2785 
Kwak, M. K. \& Han, S. B. (2007). Free Vibration Analysis of Rectangular Plate with a Hole by means of Independent Coordinate Coupling Method. Journal of Sound and Vibration, Vol. 306, (12-30), ISSN0022-460X

Kwak, M. K. \& Song, M. H. (2007). Free Vibration Analysis of Rectangular Plate with Multiple Rectangular Cutouts by Independent Coordinate coupling Method. Transactions of the Korean Society for Noise and Vibration Engineering, Vol. 17, No. 9, (881-887), ISSN1598-2785

Kwak, M. K. \& Song, M. H. (2007). Free Vibration Analysis of Rectangular Plate with Multiple Circular Cutouts by Independent Coordinate Coupling Method. Transactions of the Korean Society for Noise and Vibration Engineering, Vol. 17, No. 11, (1086-1092), ISSN1598-2785

Lam, K. Y.; Hung, K. C. \& Chow, S. T. (1989). Vibration Analysis of Plates with Cut-outs by the Modified Rayleigh-Ritz Method. Applied Acoustics, Vol. 28, (49-60), ISSN0003$682 X$

Lam, K. Y. \& Hung, K. C. (1990). Vibration Study on Plates with Stiffened Openings Using Orthogonal Polynomials and Partitioning Method. Computers and Structures, Vol. 37, (295-301), ISSN0045-7949

Laura, P. A. A.; Romanelli, E. \& Rossi, R. E. (1997). Transverse Vibrations of SimplySupported Rectangular Plates with Rectangular Cutouts. Journal of Sound and Vibration, Vol. 202, No. 2, (275-283), ISSN0022-460X

Laura, P. A. A.; Masia, U. \& Avalos, D. R. (2006). Small Amplitude, Transverse Vibrations of Circular Plates Elastically Restrained Against Rotation with an Eccentric Circular Perforation with a Free Edge. Journal of Sound and Vibration, Vol.292, (1004 1010), ISSN0022-460X

Lee, H.S. \& Kim, K.C. (1984). Transverse Vibration of Rectangular Plates Having an Inner Cutout in Water. Journal of the Society of Naval Architects of Korea, Vol. 21, No. 1, (2134), ISSN1225-1143

Lee, W. M.; Chen, J. T. \& Lee, Y. T. (2007). Free Vibration analysis of Circular Plates with Multiple Circular Holes using Indirect BIEMs. Journal of Sound and Vibration, Vol.304, (811-830), ISSN0022-460X

Lee, Y.-S. \& Lee, Y.-B. (1994). Free Vibration Analysis of 4 Edges clamped, Isotropic Square Plates with 2 Collinear Circular Holes. Transactions of Korean Society for Noise and Vibration Engineering, Vol.4, No.3, (283-293), ISSN1598-2785

Leissa, A. W. (1993). Vibration of Plates, Acoustical Society of America, New York.

Lin, W. H. (1982). Free Transverse Vibrations of Uniform Circular Plates and Membranes with Eccentric Holes. Journal of Sound and Vibration, Vol.81, No.3, (425 435), ISSN0022-460X

Monahan, L. J.; Nemergut, P. J. \& Maddux, G. E. (1970). Natural Frequencies and Mode Shapes of Plates with Interior Cut-outs. The Shock and Vibration Bulletin, Vol. 41, (3749)

Nagaya, K. (1951). Transverse Vibration of a Plate having an Eccentric Inner Boundary. Journal of Applied Mechanics, Vol.18, No.3, (1031 1036), ISSN0021-8936

Nagaya, K. (1980). Transverse Vibration of a Rectangular Plate with an Eccentric Circular Inner Boundary. Int. J. Solids Structures, Vol. 16, (1007-1016), ISSN0020-7683 
Paramasivam, P. (1973). Free Vibration of Square Plates with Square Opening. Journal of Sound and Vibration, Vol. 30, (173-178), ISSN0022-460X

Rajamani, A. \& Prabhakaran, R. (1977). Dynamic Response of Composite Plates with Cutouts, Part I: Simply-Supported Plates. Journal of Sound and Vibration, Vol. 54, (549564), ISSN0022-460X

Rajamani, A. \& Prabhakaran, R. (1977). Dynamic Response of Composite Plates with Cutouts, Part II: Clamped-Clamped Plates. Journal of Sound and Vibration, Vol. 54, (565576), ISSN0022-460X

Sakiyama, T.; Huang, M.; Matsuda, H. \& Morita, C. (2003). Free Vibration of Orthotropic Square Plates with a Square Hole. Journal of Sound and Vibration, vol. 259, No. 1, (6380), ISSN0022-460X

Takahashi, S. (1958). Vibration of Rectangular Plates With Circular Holes. Bulletin of JSME, Vol. 1, No. 4, (380-385), ISSN0021-3764

Zhong, H. \& Yu, T. (2007). Flexural Vibration Analysis of an Eccentric Annular Mindlin Plate. Archive of Applied Mechanics, Vol.77, (185 195), ISSN0939-1533 


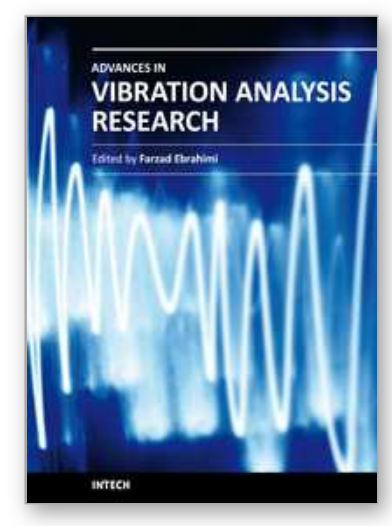

\author{
Advances in Vibration Analysis Research \\ Edited by Dr. Farzad Ebrahimi
}

ISBN 978-953-307-209-8

Hard cover, 456 pages

Publisher InTech

Published online 04, April, 2011

Published in print edition April, 2011

Vibrations are extremely important in all areas of human activities, for all sciences, technologies and industrial applications. Sometimes these Vibrations are useful but other times they are undesirable. In any case, understanding and analysis of vibrations are crucial. This book reports on the state of the art research and development findings on this very broad matter through 22 original and innovative research studies exhibiting various investigation directions. The present book is a result of contributions of experts from international scientific community working in different aspects of vibration analysis. The text is addressed not only to researchers, but also to professional engineers, students and other experts in a variety of disciplines, both academic and industrial seeking to gain a better understanding of what has been done in the field recently, and what kind of open problems are in this area.

\title{
How to reference
}

In order to correctly reference this scholarly work, feel free to copy and paste the following:

Moon Kyu Kwak and Seok Heo (2011). Independent Coordinate Coupling Method for Free Vibration Analysis of a Plate With Holes, Advances in Vibration Analysis Research, Dr. Farzad Ebrahimi (Ed.), ISBN: 978-953307-209-8, InTech, Available from: http://www.intechopen.com/books/advances-in-vibration-analysisresearch/independent-coordinate-coupling-method-for-free-vibration-analysis-of-a-plate-with-holes

\section{INTECH}

open science | open minds

\author{
InTech Europe \\ University Campus STeP Ri \\ Slavka Krautzeka 83/A \\ 51000 Rijeka, Croatia \\ Phone: +385 (51) 770447 \\ Fax: +385 (51) 686166 \\ www.intechopen.com
}

\author{
InTech China \\ Unit 405, Office Block, Hotel Equatorial Shanghai \\ No.65, Yan An Road (West), Shanghai, 200040, China \\ 中国上海市延安西路65号上海国际贵都大饭店办公楼405单元 \\ Phone: +86-21-62489820 \\ Fax: +86-21-62489821
}


(C) 2011 The Author(s). Licensee IntechOpen. This chapter is distributed under the terms of the Creative Commons Attribution-NonCommercialShareAlike-3.0 License, which permits use, distribution and reproduction for non-commercial purposes, provided the original is properly cited and derivative works building on this content are distributed under the same license. 